\title{
Reducing Various Generation Problems Based on Health Assessment for Mental Health in the Manufacturing Industry -Establishment of Organizational Assessment Management System-
}

\author{
HIROHISA SAKAI \\ Toyota Motor Corporation \\ 1, Motomachi, Toyota-shi, Aichi-ken, 471-8573, Japan \\ JAPAN
}

\begin{abstract}
At present, the labor force lowering by the mental health of the employee in the manufacturing site is remarkable, and it is noticed as a factor which causes labor accident, traffic accident, and social problem action. In addition, the Ministry of Health, Labor and Welfare in Japan will emphasize mental health elimination as an occupational health measure, and the government intends to submit a mandate for stress checks by doctors or health technicians within this ordinary Diet. On the other hand, the company is also promoting prevention activities focusing on "organization" by activating people's cooperative behavior from the health support activity corresponding to the analysis of conventional individual features from the viewpoint of occupational health. In this paper, the health assessment "Occupational Stress Survey" identified that the supervisor support in the organization contributes greatly and constructed "Organizational Assessment System" which takes countermeasures from both sides of human resources and occupational health of the organization in the manufacturing industry.
\end{abstract}

Key-Words: - Mental Health, Health Assessment, Organizational Assessment System

Received: May 20, 2020. Revised: June 30, 2020. Accepted: March 10, 2021. Published: March 20, 2021.

\section{Introduction}

At present, the decrease in labor force due to the mental health of employees at the manufacturing site is remarkable, and the percentage of workers who feel strong stress in the workplace and work is more than $60 \%$, and the number of occupational accidents accredited for the reason of mental disability is increasing for the third consecutive year, and the improvement of the working environment is an urgent issue. Against this backdrop, the Ministry of Health, Labor and Welfare in Japan has submitted a stress check requirement by a doctor or a healthcare technician (Bill 6 to revise part of the Occupational Safety and Health Law) within the ordinary Diet on March 13, 2014 (Labor Standards Public Relations) in order to ensure that workers are aware of the health status of workers before they suffer mental trouble in order to enhance and strengthen mental health measures in the workplace.

On the other hand, the company is also promoting prevention activities focusing on "organization" by activating people's cooperative behavior from the health support activity corresponding to the analysis of conventional individual features from the viewpoint of occupational health. Otsuka et al. have found a significant correlation between support from boss and colleagues within the organization and safety considerations and movements in the workplace, suggesting that the support of one of boss and colleagues is important for the execution of worker safety actions [1]. Organizations with rich social characteristics such as "trust," "norms," and "networks" have high levels of health and productivity, significant economic growth, and low levels of crime by stimulating people's cooperative behavior [2]-[5].

In this paper, it was found by health assessment "Occupational Stress Survey" that the supervisor support in the organization contributed greatly using the occupational stress simplified survey sheet (Ministry of Health, Labor and Welfare, 2000) by the Ministry of Health, Labor and Welfare in Japan. Based on the results, the author has constructed an "Organizational Assessment Management System" to take countermeasures from the viewpoints of personnel and occupational health of the organization at automobile company Toyota in the manufacturing industry [6][7].

\section{Objects and Methods of Health Assessment "Occupational Stress Survey"}




\subsection{Subject to the Occupational Stress Survey}

The author has surveyed 2772 workers working at Toyota. The first survey was conducted from October to November 2012, and the second survey was conducted using data on the average value for each group organized twice: February to March 2013, to verify whether 178 groups of disasters, traffic accidents, and social problem behavior that occurred in 2011 and 2012 can be identified.

\subsection{Method of "Occupational Stress Survey"}

The Occupational Stress Simplified Survey Sheet (Ministry of Labor, 2000) as shown in Figure 1 is a research result of the Health, Labor and Welfare Science Research Group of the Ministry of Health, Labor and Welfare in Japan was developed to explore ways of use, evaluation, and taking countermeasures in non-health sectors such as management policies, personnel systems, workplace management, and corporate climate outside the fields of health management, which can be used in workplaces of all types of industries. Three measures of workplace climate, labor force and productivity, and psychological stress can be measured from the survey results. First, the scale of the workplace climate is composed of 7 lower scales of "Quantitative burden" (3 items), "Qualitative burden" (3 items), "Control" (3 items), "Support of the boss" (3 items), "Support of colleagues" (3 items), "Sense of unity" (3 items), and "Sense of stability" ( 3 items), and a total of 21 items. Next, the scale of labor force and productivity is composed of three subordinate scales of "Performance: body" (4 items), "Performance: spirit" (3 items), and "Vigor" (3 items), and a total of 10 items. Finally, the scale of psychological stress is composed of 2 subscales of "Sense of fear" (3 items) and "Depressive feeling" (6 items), and a total of 9 items. The response methods are 4 cases from "1: Yes" to "4: Differ".

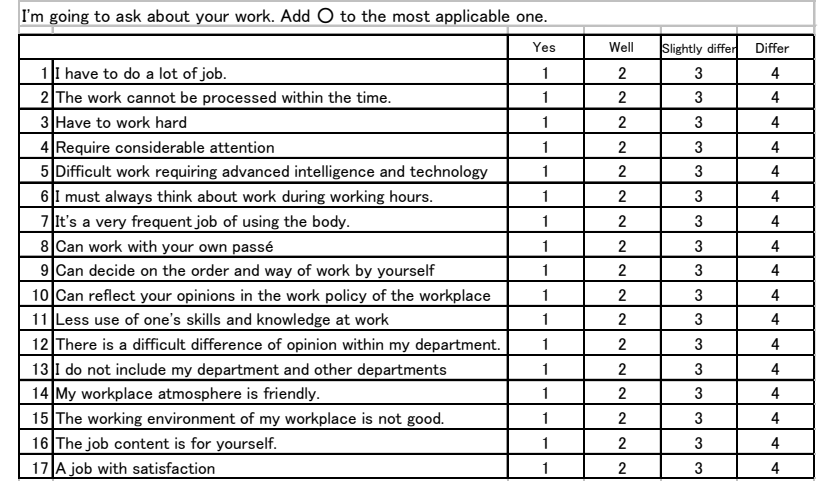

Fig.1 Simple Occupational Stress Survey

\subsection{Aggregation of results of "Occupational Stress Survey"}

Of the 40 individual questionnaire results mentioned above, 17 items as shown in Figure 2 were selected as items related to the organization. Aggregated for each set of organization using this personal data, and the averaged value is the data of each set. At the beginning of the analysis, a summary of the questionnaire results is shown in Figure 2. This is the data distribution of each set summarized in the evaluation scale of 17 items mentioned above, and the vertical axis shows the number of sets, and the horizontal axis shows the score, and the higher the number, the more positive the answer.

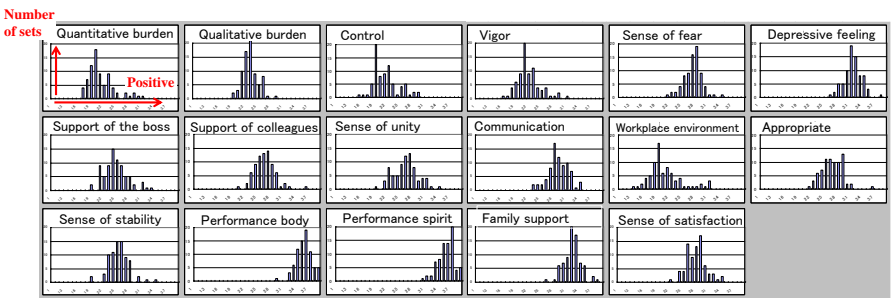

Fig.2 Distribution of Set Data-17 Evaluation Scales-

\subsection{Analysis of Occupational Stress Survey Results}

Using the data of average value for every braid which composes this organization, whether it can distinguish the set which generates 178 problems of disasters, traffic accidents, and social problem actions which arose in 2011 and 2012 (1) discriminant analysis, and (2) quantification II type were verified. First, by (1) discriminant analysis of Figure 3, a set with a problem positive value, a set without problem is determined as a negative value, the pairs with a problem is shown in white, the pairs without blue. 


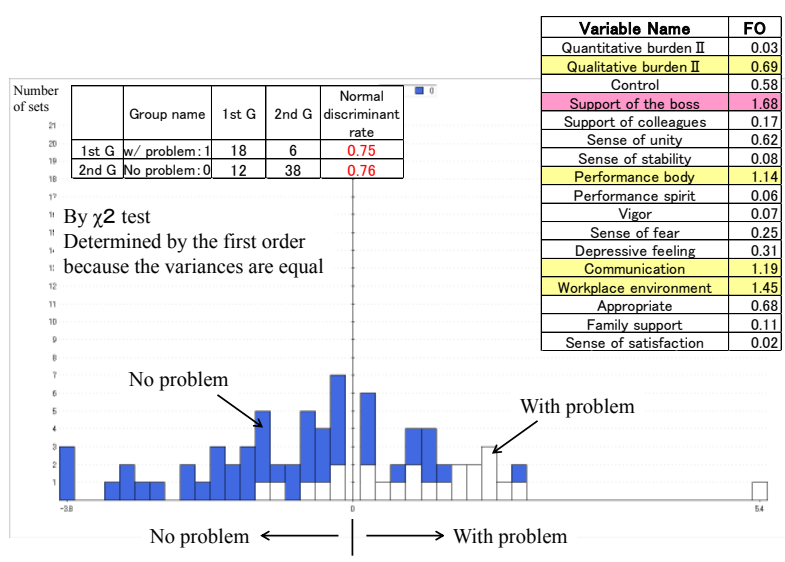

(1) Judgement by discriminant analysis

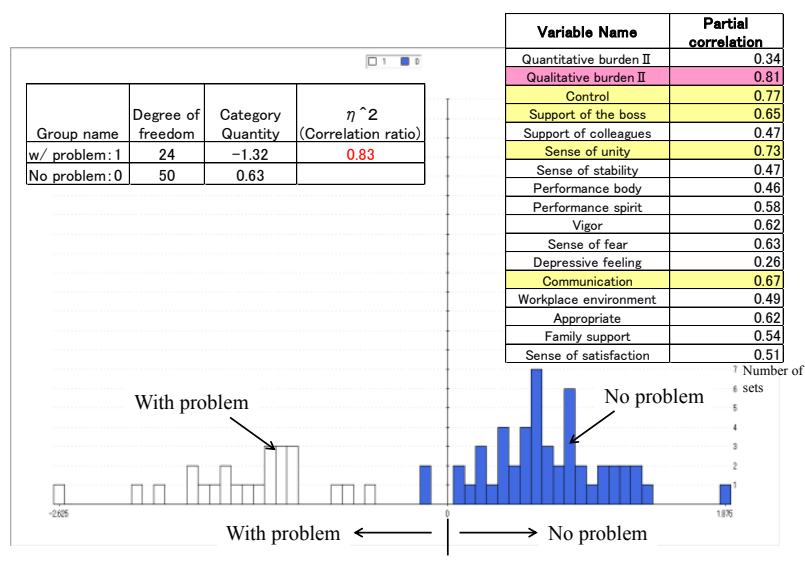

(2) Judgement according to Quantification type II

Fig. 3 Determination of sets of workplace problems

As a result of analyzing the data, both of them had the right discriminant rate of about 0.75 . By this, it can be said that there is a causal relation between the evaluation scale of the occupational stress survey and the workplace problem. Among the 17 evaluation scales, "Support of the boss" contributed most, followed by "Workplace environment" and "Communication" were found to have a large impact. Next, the correlation ratio of 0.83 was obtained even in (2) Quantification type II of Figure 3 [8]. Among them, "Qualitative burden" contributed the most, and subsequently, "Control" and "Sense of unity" were found to be largely influenced.

By these, the causal relation between the evaluation scale of the occupational stress simplified survey and the workplace problem was recognized. The top five items with large impact in the former analysis are "Support of the boss", "Workplace environment", "Communication", "Performance: body", and "Qualitative burden", and the latter are "Qualitative burden", "Control of work", "Sense of unity", "Communication", and "Support of the boss". Therefore, three items common to both results are "Support of the boss", "Communication," and "Qualitative burden".

In addition, positioning will be carried out to analyze the degree of impact on the seven items of "Support of the boss", "Workplace environment", "Communication", "Performance: spirit", "Qualitative burden", "Control", and "Sense of unity" obtained by the analysis of both.

The distribution of the eigenvectors of the seven items described above is shown in (1) Principal Component Analysis as shown in Figure 4. The first principal component of the horizontal axis indicates the magnitude of stress, and the stress held toward the right is small. On the other hand, the second principal component on the vertical axis indicates the effect of the work itself in the + direction, and the -(minus) direction indicates the effect of the environment around such as support and communication. The principal components analysis results for each set are

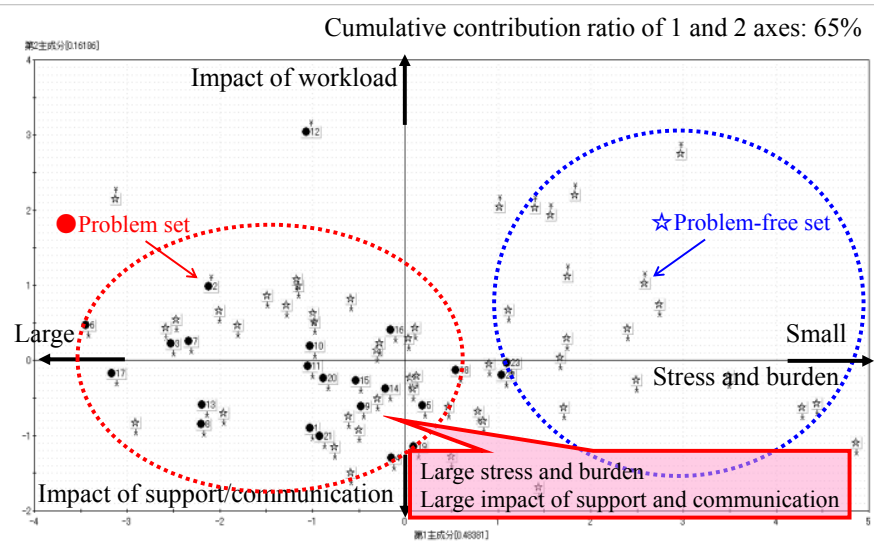

(1) Principal Component Analysis

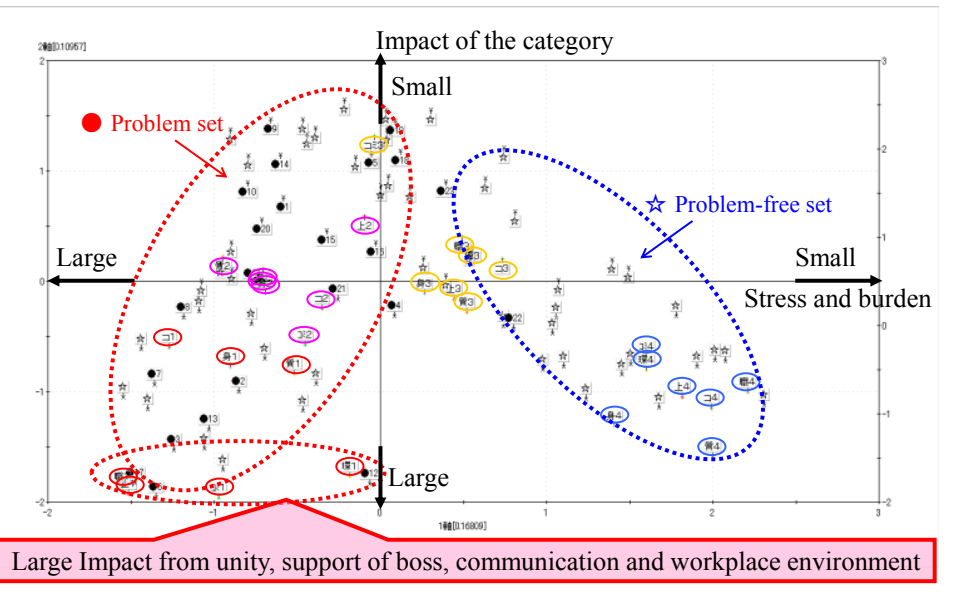

(2) Quantification type III

Fig. 4 Principal Component Analysis and Quantification type III Results for Each Pair 
then plotted. Marks indicate groups with various problems and groups without is marks, and many of marks show a great deal of stress and burden, and the effect is due to support and communication.

Similarly, the results of Quantification type III of each set are plotted in (2) as shown in Figure 4 [8].

OMarks indicate groups with various problems and represent groups without is marks, and many of marks show a large degree of influence due to "Sense of unity", "Support of the boss", "Communication", and "Work environment", and these factors can be said to greatly influence stress.

As a result of examining a set which generates various problems of disasters, traffic accidents, and social problem actions using the data of average value for each set which composes this organization, it was found that the group (workshop) in which various problems arose has a large degree of stress and burden, and that the stress is more influenced by the environments such as support of the boss and communication than the effect of the business itself such as qualitative work.

\section{Organizational Assessment System: Creation of "TEAMAS"}

\subsection{Aim of "TEAMAS" Innovation}

According to the results described above, there are limitations in the health support activities that respond by analyzing the characteristics of individual until now, and it is important to optimize the proportion of management supervisors who organize organizations for individual members, and to support supervisors and care for health risks as workplaces for those who have a sense of burden and isolation of work.

\subsection{Configuration of "TEAMAS"}

Therefore, "TEAMAS (TEAM Asses-Management System)" which grasps the necessity of the care as a workplace and optimizes the workplace operation condition for the person who has the sense of burden and isolation of the job was devised. The system diagram of "TEAMAS" is shown in Figure 5, and it has a system for optimization of workplace operation based on health-risk care and support of boss. Features of the system are as follows: Regarding organizational organization, review of the control span, optimization of the ratio outside the line, and introduction of the group leader (GL) assistant are carried out in order to embody care management in the workplace, and improvement of communication is attempted by rationalization.

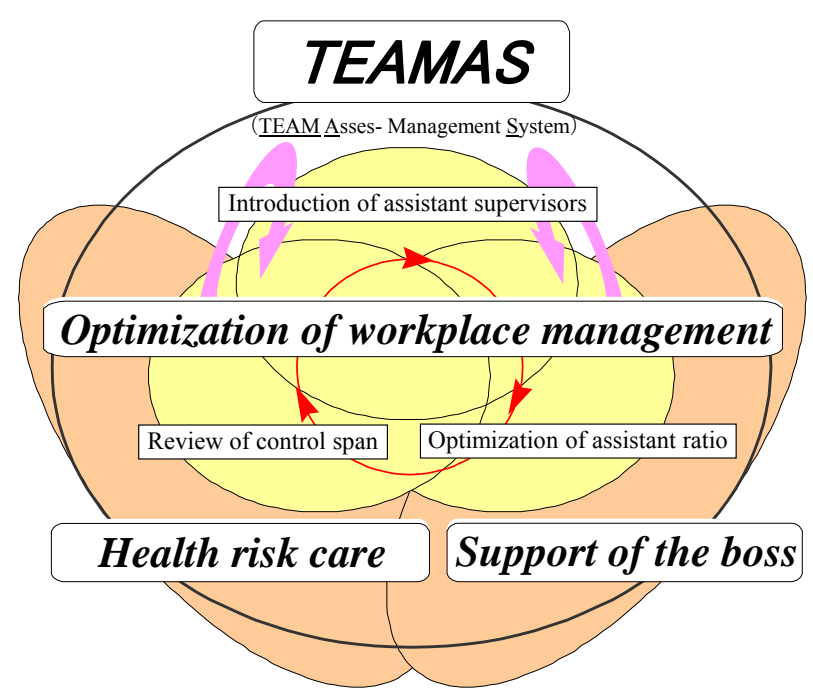

Fig.5 System Diagram of "TEAMAS"

\section{Organizational Assessment System: "TEAMAS" Study Case Study}

The results of the percentage of assistants supporting the line operator after the introduction of the system described above, the control span of the set, and the management span of the staff and the ratio of the set generated various problems are shown in Figure 6.

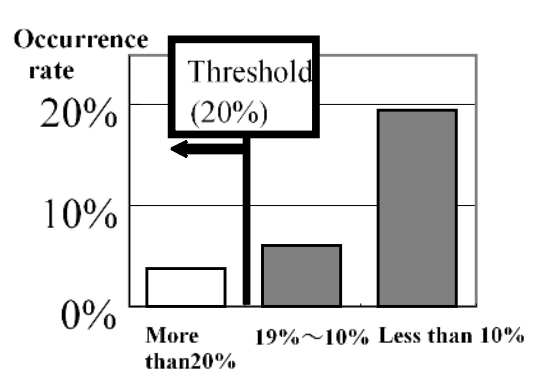

(1) Assistant ratio

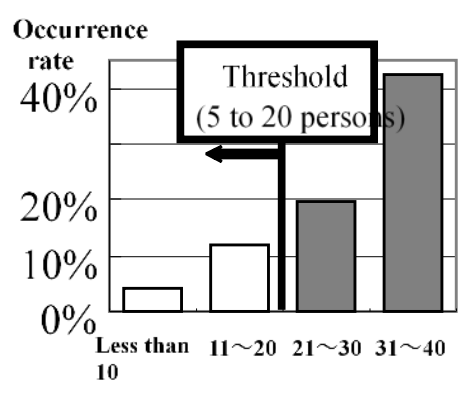

(2) Set control span

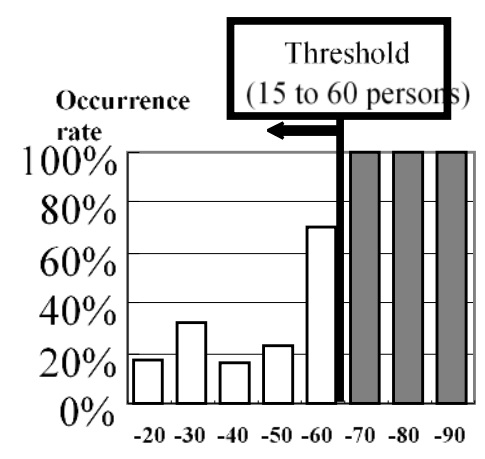

(3) Control span

Fig. 6 Percentage of sets that caused problems with assistant ratios and line spans 


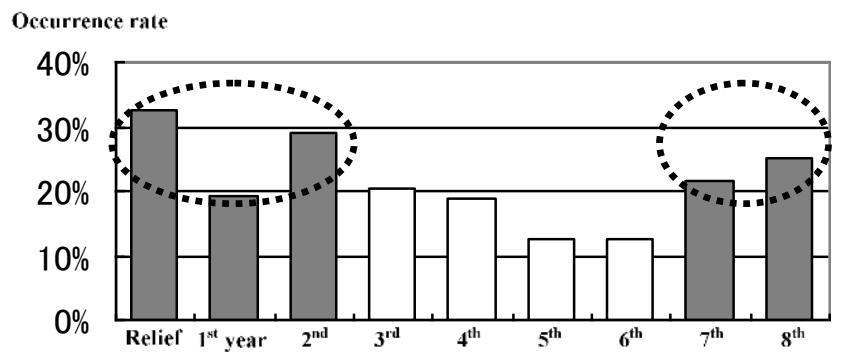

(1) Qualified Chief Experience

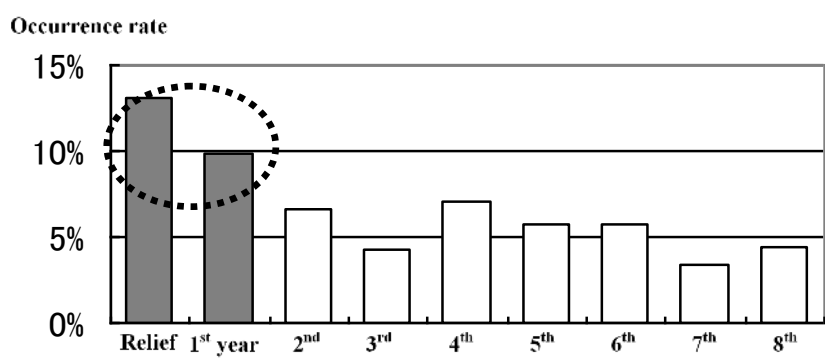

(2) Qualified Team Leader Experience

Fig. 7 Percentage of sets that caused various problems according to the number of years of management qualification experience

The verification results showed that (1) the assistant ratio was $20 \%$, (2) the set management span was 5 to 20 persons, and (3) the supervisory span was 15 to 60 persons was the threshold value of rapid increase in various problems.

Figure 7 shows the number of years of qualified experience for the supervisor and the percentage of sets of problems that occurred. It was found that the occurrence rate from the workplaces of the qualified chief engineer with short experience years and veteran qualified chief engineer was high, and the occurrence rate from the workplaces with short experience years including representatives was also high with respect to the qualified team leader experience engineer. Then, the veteran chief was made to recognize the role recognition again, and the stress of the manager was reduced in the model set which introduced the management supervisor assistant, and the occurrence of various problems was reduced by half.

The higher the health risk, the $30 \%$ higher the problem rate. Estimate the problem rate as shown in Figure 8, because the health risk worsens in workplaces without "Vigor" or "Sense of unity" and leads to problems. In order to reduce the health risk, the occurrence of various problems was finally reduced by less than $60 \%$ by enhancing the support of the boss for a group with a high problem occurrence rate.
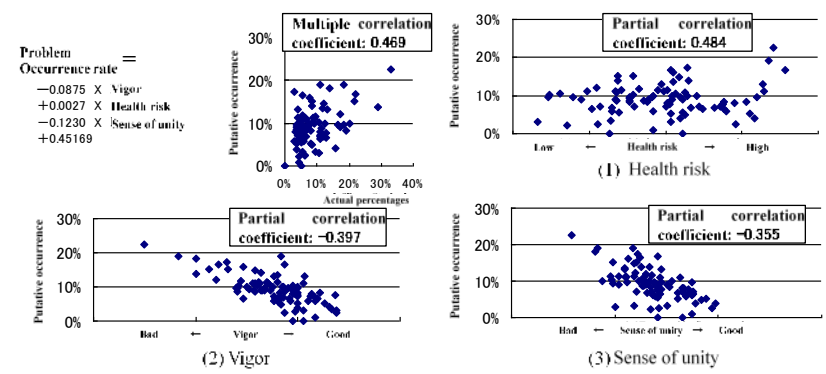

Fig.8 Estimation of Problem Occurrence Rate ("Health risk", "Vigor", and "Sense of unity")
Finally, Figure 9 shows the results of the survey conducted again after the introduction of the aforementioned system. Various issues are generated, and the plotting after the system introduction of the set is indicated by $\boldsymbol{\Delta}$ mark. The results of the tests showed that the graph was shifted to the lower right of the graph in many sets, indicating that stress was reduced, and it was noticeable that the direction was greatly improved, especially in the model set in which GL assistant was introduced. Finally, the occurrence of various problems is also

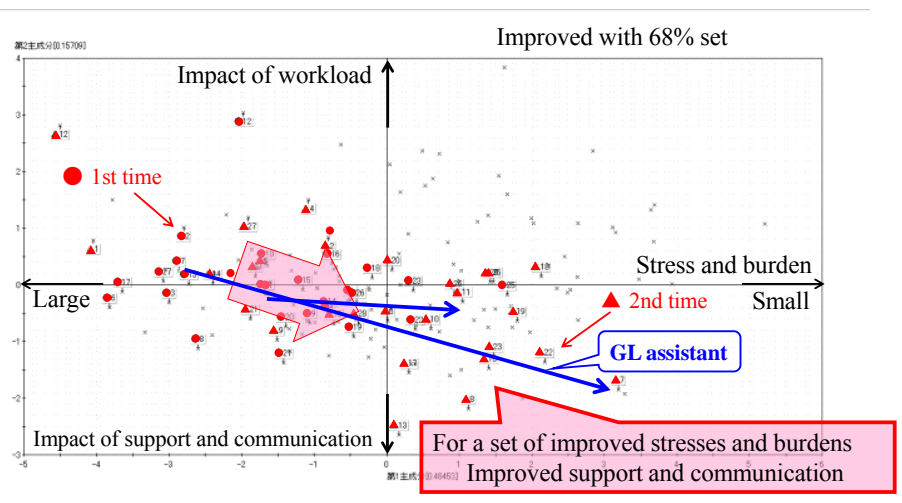

reduced.

Fig.9 Verification of stress changes in each set

\section{Conclusion}

The author has proposed the "TEAMAS" which grasps the necessity of the care as a workplace and optimizes the workplace operation condition for the person who has the sense of burden and isolation of the work and demonstrated the effectiveness through the demonstration example of automobile company Toyota in the manufacturing industry.

\section{References:}

[1] Yasumasa Otsuka and Ayako Suzuki, Associations between support from bosses and colleagues and workplace safety behaviour, 
Journal of Japan Society for Occupational Health, Vol.48, 2006, pp. 650.

[2] Putnam, Robert D., Making democracy work: Civic tradition in modern Italy, Princeton, N.J.: Princeton University Press, 1993, pp.258.

[3] Katsunori Kondo, Health Difference Society: What corrupts heart and health?, Igaku-Shoin, 2005.

[4] Teruichi Shimotsumi (2005), "Manual for understanding the current status of stress using a simple occupational stress survey form - For more effective improvement measures for the workplace environment", Ministry of Health, Labour and Welfare Science and Research Subsidies General Research on Occupational Safety and Health (Research on Mental Health Measures by Improving Workplace Environments, etc.).

[5] Hiroshi Kai (2019), "Issues regarding the implementation contents of Health and Safety Management activities at workplace with occurrence of work-related accident", National Institute of Occupational Safety and Health, Japan, Vol. 12, No. 1, pp. 13-24.

[6] Hirohisa Sakai, Kakuro Amasaka (2014), "Reduction Various Generation Problems Based on Health Assessment for Mental Health in the Manufacturing Industry -Establishment of Organizational Assessment Management System-", 7th Japanese Operations Management and Strategy Association Conference, Takushoku University, pp.1-4.

[7] Hirohisa Sakai, Kakuro Amasaka (2015), "Reduction Various Generation Problems Based on Health Assessment for Mental Health in the Manufacturing Industry (2) -Utilization of Organizational Assessment Management System-", 7th Japanese Operations Management and Strategy Association Conference, Gakushuin University, pp.1-4.

[8] Chikio Hayashi (1993), Quantitation - Theory and Method (Statistical Library), Asakura Publishing Co., Ltd.

\section{Appendix}

Occupational Stress simple Survey Form: Ministry of Labor "Research Group on Prevention of WorkRelated Diseases"-In the Stress Measurement Research Group, a questionnaire on many existing stresses was examined under consignment from the Ministry of Labor, and a simplified questionnaire on occupational stress with high reliability and validity that can be easily measured and evaluated in the field was developed. And, this paper prepares a manual for grasping the present state of stress using an occupational stress simplified survey form as a result of "research on mental health countermeasures by improvement of workplace environment, etc." of the Health, Labor and Welfare Science Research Subsidy General Research Project for Occupational Safety and Health in 2002-2004.

\section{Creative Commons Attribution License 4.0 (Attribution 4.0 International, CC BY 4.0)}

This article is published under the terms of the Creative Commons Attribution License 4.0 https://creativecommons.org/licenses/by/4.0/deed.en_US 